\title{
Cumulative Perturbations Affecting a Spacecraft on a Mars Equatorial Orbit from the Waxing and Waning of the Polar Caps of the Planet
}

\author{
Jean-Pierre Barriot \\ Geodesy Observatory of Tahiti, University of French Polynesia, Faa'a, Tahiti \\ Email: jean-pierre.barriot@upf.pf
}

Received 5 December 2015; accepted 19 December 2015; published 23 December 2015

Copyright (C) 2015 by author and OALib.

This work is licensed under the Creative Commons Attribution International License (CC BY). http://creativecommons.org/licenses/by/4.0/

\section{(c) (i) Open Access}

\begin{abstract}
We demonstrate in this paper that periodic variations of the $J_{2}$ gravity coefficient of a planet induce small cumulative perturbations on a given family of circular equatorial orbits, and that these perturbations could be measurable with current radiosciences technology. For this purpose, we first consider a Poincaré expansion of the Newtonian equations of motion. Then, by using Floquet's theory, we demonstrate that, unlike the excitation mechanism, the perturbations are nonperiodic, and that the orbit is not "stable" in the long-term, with perturbations growing exponentially. We give the full theory and an application to the case of planet Mars.
\end{abstract}

\section{Keywords}

Mars' Length-of-Day, Orbit Perturbations, Floquet's Theorem

Subject Areas: Geodesy, Geophysics

\section{Rationale}

Chao and Rubincam [1] demonstrated that the $J_{2}$ harmonic moment of Mars is subject to large annual variation as about one quarter of the $\mathrm{CO}_{2}$ atmosphere condenses during winters at the poles, and sublimes during summers (see Figure 1), with

$$
J_{2}(t)=J_{2}^{o}+\Delta \cos (\omega t+\varpi)
$$

How to cite this paper: Barriot, J.-P. (2015) Cumulative Perturbations Affecting a Spacecraft on a Mars Equatorial Orbit from the Waxing and Waning of the Polar Caps of the Planet. Open Access Library Journal, 2: e2272.

http://dx.doi.org/10.4236/oalib.1102272 


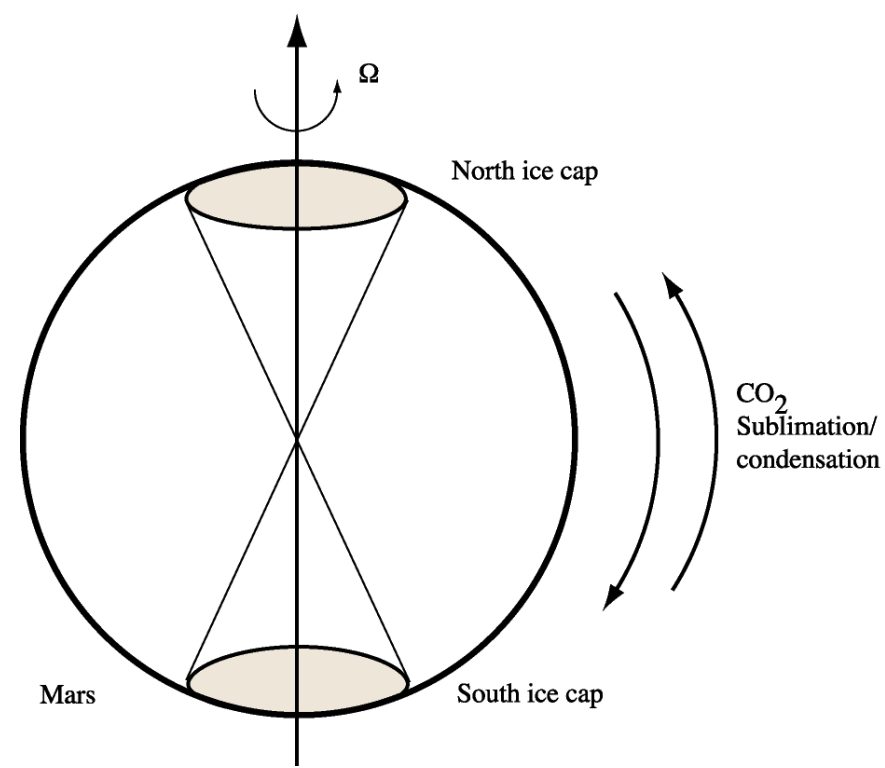

Figure 1. The hourglass model of the sublimation/condensation mechanism on Mars. No terrestrial phenomenon is known to produce a mass redistribution of such a magnitude over a year.

where $\Delta=-\frac{3}{2} \frac{C_{0}}{M R^{2}} \frac{\sigma \omega}{\Omega_{0}} ; \varpi$ is an arbitrary phase; $\sigma$ is the annual length-of-day variation; $C_{0}$ is the Mars mean polar inertial moment; $\Omega_{0}$ is the mean Mars angular rotation velocity; $\omega$ is the mean Mars angular orbital velocity; $R$ is the Mars radius; and $M$ is the planet's mass. Similar variations, but of a lesser amplitude, are also observed on the Earth. In this paper, we show that these small variations can end up in cumulative perturbations on selected families of orbits. This work can be easily extended to semi-annual perturbations and larger degree and order gravity coefficients [2]. The only restriction is that these perturbations must have commensurate periods.

\section{Orbital Mechanics}

With respect to a given inertial cartesian coordinate frame, the Newtonian equations of motion of a space probe orbiting a planet are

$$
\begin{aligned}
& \ddot{x}=-\mu \frac{x}{r^{3}}-J x \frac{x^{2}+y^{2}-4 z^{2}}{r^{7}}+\varepsilon \frac{\partial U}{\partial x}+\eta f_{x} \\
& \ddot{y}=-\mu \frac{y}{r^{3}}-J y \frac{x^{2}+y^{2}-4 z^{2}}{r^{7}}+\varepsilon \frac{\partial U}{\partial y}+\eta f_{y} \\
& \ddot{z}=-\mu \frac{z}{r^{3}}-J z \frac{3 x^{2}+3 y^{2}-2 z^{2}}{r^{7}}+\varepsilon \frac{\partial U}{\partial z}+\eta f_{z}
\end{aligned}
$$

where $r=\left(x^{2}+y^{2}+z^{2}\right)^{1 / 2}, \mu$ is the gravitational constant of Mars, $J=\frac{3}{2} \mu R^{2} J_{2}, \varepsilon \nabla U$ represents the remaining part of the gravity field (without the $J_{2}$ term), and the $\eta \boldsymbol{f}$ term summarizes all the other forces like atmospheric drag (the main contributor for low orbits), sun tidal acceleration, solar pressure, relativistic corrections, etc. We use the notation $\varepsilon \nabla U$, where $\varepsilon$ is a global scaling factor [3], to emphasize the fact that these forces are of small amplitudes with respect to the central and $J_{2}$ terms. The aims of the notation $\eta \boldsymbol{f}$ is similar.

We now consider a probe orbiting the planet on a high altitude (i.e. with $\varepsilon \approx 0$ ) near equatorial orbit (i.e. with $z \approx 0$ ) in order to avoid the atmospheric drag (i.e. with $\eta \approx 0$ ). Up to the first order in $z$, this leads to 


$$
\begin{aligned}
& \ddot{x}=-\mu \frac{x}{d^{3}}-J \frac{x}{d^{5}} \\
& \ddot{y}=-\mu \frac{y}{d^{3}}-J \frac{y}{d^{5}} \\
& \ddot{z}=-\mu \frac{z}{d^{3}}-3 J \frac{z}{d^{5}}
\end{aligned}
$$

where $d=\left(x^{2}+y^{2}\right)^{1 / 2}, \quad z \ll d$.

It is clear that the last equation is decoupled from the first two ones. The solution of the third one corresponds to an oscillation with respect to the mean orbital plane, of no interest for the following discussion.

If we switch to cylindrical coordinates $d, \varphi, z$, where $x=d \cos \varphi$ and $y=d \sin \varphi$, we obtain

$$
\begin{aligned}
& \ddot{d}=d \dot{\varphi}^{2}-\frac{\mu}{d^{2}}-\frac{J}{d^{4}} \\
& \frac{\mathrm{d}}{\mathrm{d} t}\left(d^{2} \dot{\varphi}\right)=0 \Leftrightarrow d^{2} \dot{\varphi}=h_{0} .
\end{aligned}
$$

The constant $h_{0}$ can be identified as an angular momentum.

Jezewski [4] [5] demonstrated that if $\Delta=0$, i.e. if $J=J^{0}=\frac{3}{2} \mu R^{2} J_{2}^{0}$, then Equation (1') admits a solution in terms of ellipsoidal functions as

$$
\begin{aligned}
& u(\varphi)=c+(b-c) s^{2}\left(\frac{\varphi-\varphi_{c}}{\gamma g}\right) \\
& t(\varphi)=t_{c}+\int_{\theta=\varphi_{c}}^{\varphi} \frac{\mathrm{d} \theta}{u^{2}(\theta)}
\end{aligned}
$$

where $u=\frac{1}{d}$, and $\operatorname{sn}(x)$ is the Jacobi ellipsoidal sine function. The two constants $b$ and $c$ are determined by the initial conditions, and have a direct physical meaning as the inverses $b=\frac{1}{d_{p}}$ of the periapsis and apoapsis $c=\frac{1}{r_{a}}$ of the orbit. The other two constants are given by $\varphi_{c}$ and $t_{c}$. One can demonstrate that $a=\frac{1}{(b+c)}\left(\frac{3 \mu}{J^{0}}-b c\right)$, with $a>b>c$. The constant $k=\left(\frac{b-c}{a-c}\right)^{1 / 2}$ is the modulus of the Jacobian sn function, and $\gamma=h_{0} \sqrt{\frac{3}{2 J^{0}}}, g=\frac{2}{\sqrt{a-c}}$. The functions $u$ and $\varphi$, viewed as a function of $t$ are periodic, but with different periods, and define an "ellipsis" with an apse line slowly rotating in the equatorial plane, with a period $F(\pi / 2 ; k)$ (complete elliptic integral of the first kind). A close value of the angular velocity of the apse line can be deduced from the usual Laplace equations by summing the $J_{2}^{0}$ secular drifts $\dot{\Omega}$ of the line of node and of the line of apsides $\dot{\omega}$ for a non equatorial orbit of inclination $i$ and semi-major axis $a$ as

$\dot{\Omega}=-\frac{3}{2} n \frac{J_{2}^{0} R^{2} \cos i}{\left(1-e^{2}\right)^{2} a^{2}}$ and $\dot{\omega}=-\frac{3}{4} n \frac{J_{2}^{0} R^{2}\left(1-5 \cos ^{2} i\right)}{\left(1-e^{2}\right)^{2} a^{2}}, \Omega+\omega$ being a continuous quantity when $i \rightarrow 0$, with limit $\dot{\Omega}+\dot{\omega}=\frac{3}{2} n \frac{J_{2}^{0} R^{2}}{\left(1-e^{2}\right)^{2} a^{2}}$.

The Hamiltonian of the unperturbed motion is given by

$$
H_{e}=\frac{1}{2} \dot{d}^{2}+\frac{1}{2} d^{2} \dot{\varphi}^{2}-\frac{\mu}{d}-\frac{1}{3} \frac{J_{0}}{d^{3}} .
$$


If $\Delta \neq 0$, Poincaré's theorem [6] asserts that the equations of motion can be developed with $J=J_{0}+\Delta \phi$ as

$$
\begin{aligned}
& d=d_{0}+\Delta d_{1}+\Delta^{2} d_{2}+\cdots \\
& \varphi=\varphi_{0}+\Delta \varphi_{1}+\Delta^{2} \varphi_{2}+\cdots \\
& z=z_{0}+\Delta z_{1}+\Delta^{2} z_{2}+\cdots
\end{aligned}
$$

where $d_{1}, \varphi_{1}, z_{1}, d_{2}, \varphi_{2}, z_{2} \cdots$ satisfy differential equations with null initial conditions. These differential equations are determined by plugging Equation (3) into Equation (1'), and equating the powers of $\Delta$.

For the first order, after some uninteresting algebra, we arrive at

$$
\begin{gathered}
\ddot{d}_{1}=\alpha d_{1}+\beta \dot{\varphi}_{1}+\gamma \phi \\
\ddot{\varphi}_{1}=\delta d_{1}+\tau \dot{d}_{1}+\lambda \dot{\varphi}_{1} \\
\alpha=\dot{\varphi}_{0}^{2}+2 \frac{\mu}{d_{0}^{3}}+4 \frac{J_{0}}{d_{0}^{5}}, \quad \beta=2 d_{0} \dot{\varphi}_{0}, \quad \gamma=-\frac{1}{d_{0}^{4}} \\
\delta=2 \frac{\dot{d}_{0} \dot{\varphi}_{0}}{d_{0}^{2}}, \quad \tau=-2 \frac{\dot{\varphi}_{0}}{d_{0}}, \quad \lambda=-2 \frac{\dot{d}_{0}}{d_{0}} .
\end{gathered}
$$

This system can be rewritten as a first order system by using the usual trick $p=\dot{u}, q=\dot{v}$. This gives

$$
\left[\begin{array}{c}
\dot{u} \\
\dot{v} \\
\dot{p} \\
\dot{q}
\end{array}\right]=\left[\begin{array}{cc}
0 & I \\
M & 0
\end{array}\right]\left[\begin{array}{l}
u \\
v \\
p \\
q
\end{array}\right]+\left[\begin{array}{c}
0 \\
W
\end{array}\right]
$$

$\Leftrightarrow \dot{s}=N s+w$, with $s\left(t_{0}\right)=0$.

Similar equations can be obtained from the formalisms of Hill or Lagrange. The approach that we retained is the simplest one. Considering an equatorial circular orbit is a fundamental assumption, as it allows us to write a very simple analytical solution.

\section{Floquet's Theory}

System (5) is of Floquet's type [7], i.e. the coefficient matrix $N$ is periodic, here with a period of half an orbit. More precisely, system (5) is a two-dimensional generalization of the Mathieu's equation [8].

The solution of the system with the second member $w$ is given, as $s\left(t_{0}\right)=0$, by

$$
s(t)=\int_{t_{0}}^{t} \Pi(t, \xi) w(\xi) \mathrm{d} \xi
$$

where $\Pi$ is the solution of the homogeneous matrix system

$$
\dot{\Pi}\left(t, t_{0}\right)=N \Pi\left(t, t_{0}\right)
$$

with $\Pi\left(t_{0}, t_{0}\right)=I$ being the identity matrix. Because of uniqueness properties, we have $\Pi\left(t_{2}, t_{1}\right) \Pi\left(t_{1}, t_{0}\right)=\Pi\left(t_{2}, t_{0}\right)$, and therefore $\Pi\left(t, t_{0}\right)^{-1}=\Pi\left(t_{0}, t\right)$. As $N$ is periodic with period $T$, i.e. $N(t+k T)=N(t)$, Floquet's theorem asserts that the solutions $\Pi\left(t, t_{0}\right)$ are pseudo-periodic, i.e. that they can be written as $\Pi\left(t, t_{0}\right)=P\left(t, t_{0}\right) \exp \left(\left(t-t_{0}\right) Q\left(t_{0}\right)\right)$, where $P\left(t+T, t_{0}\right)=P\left(t, t_{0}\right)$ is periodic with $P\left(t_{0}, t_{0}\right)=I$, and $Q\left(t_{0}\right)$ is a constant characteristic of the system, with $Q\left(t_{0}+T\right)=Q\left(t_{0}\right)$. The boundedness of the solutions of systems (5) and (7) is governed by the constant matrix $C=\Pi\left(t_{0}+T, t_{0}\right)=\exp \left(T Q\left(t_{0}\right)\right)$, more precisely by its spectral radius $\rho$ (the largest eigenvalue [9]). In particular, if $\rho>1$, the solution of the homogeneous system (7) is unbounded, as well as the solution of the inhomogeneous system, unless ad'hoc (and unphysical) initial conditions are imposed [10].

\section{Long-Term Behaviour of the Orbit}

If $w(\xi)$ is periodic with the same period $T$, one can go a little farther and it can be shown (see Appendix A) 
that we have the geometrical series behavior [11]

$$
s(t+k T)=s(t)+\Pi\left(t, t_{0}\right) U^{(k)} s\left(t_{0}+T\right)
$$

with $t_{0} \leq t<t_{0}+T, k \geq 0, U^{(k)}=I+C+\cdots+C^{(k-1)}=I+C U^{(k-1)}, C=\Pi\left(t_{0}+T, t_{0}\right)$ and $U^{(0)}=0, U^{(1)}=I$.

This relation shows that the behavior of this system in the "long" term is governed by $U^{(k)}$, as $\Pi\left(t, t_{0}\right)$ is bounded for $t_{0} \leq t<t_{0}+T$. It is clear that if $U^{(k)}$ diverges, i.e. if $\rho>1$, then $s(t+k T)$ diverges too, unless $s\left(t_{0}+T\right)=0$ (and then $s(t)$ is periodic of period $T$ ). This does not mean that the physically perturbed motion is unbounded, but that the Poincaré's expansion (3) will break down at some stage. Physical bounds for the motion could probably be obtained by extending the works of Mioc and Stavinschi [12]-[14] to a variable $J_{2}$. To obtain a common period for $N$ and $w$, we just have to slightly adjust the altitude of the spacecraft, in order to have an entire number of orbital periods during a Martian year that is then becoming the common period $T$.

\section{Numerical Results and Conclusions}

Let us consider the solution for the particular case of the planet Mars and for a circular equatorial orbit. The period $T$ of a circular equatorial orbit of radius $d$ is given from (1') by $\left(\frac{2 \pi}{T}\right)^{2}=\frac{\mu}{d_{e}^{3}}+\frac{3}{2} \frac{\mu}{d_{e}^{5}} R^{2} J_{2}^{0}$.

We take the numerical values from [15] [16].

$$
\begin{gathered}
\mu=42828.376383 \mathrm{~km}^{3} / \mathrm{s}^{2}, \quad J_{2}^{0}=1.95869919367 \times 10^{-3} \quad \text { (unnormalized), } \\
C_{0} / M R^{2}=0.3662, R=3394.2 \mathrm{~km}, \Omega_{0}=7.088218111 \times 10^{-5} \mathrm{rad} / \mathrm{s}, \\
\omega=1.05857641382 \times 10^{-7} \mathrm{rad} / \mathrm{s}, \quad R \sigma=7.85 \mathrm{~m} \quad \text { (corresponding to } 477 \text { milliarcseconds). }
\end{gathered}
$$

From these values, we derive $\Delta=-1.8971 \times 10^{-9}$, i.e. a $10^{-6}$ relative variation with respect to the $J_{2}^{0}$ term.

We now consider a circular orbit well beyond the atmosphere, at an altitude of $1000.629961 \mathrm{~km}$ (semi-major axis $4394.829961 \mathrm{~km}$ ), in order to have exactly 6716 orbits/Martian year, corresponding to an orbital period of 147.266 min.

This leads to $\rho=1.002627$ for the spectral radius $\rho$ over one Martian year, from the Jordan form of the matrix $C$.

For the first year, the perturbations (norm of the differences between the perturbed and unperturbed motion) range up to $172.58 \mathrm{~m}$ in position, most of it in the along-track direction, and $122.69 \mathrm{~mm} / \mathrm{s}$ in velocity. They are measurable with state-of-the-art technology, both for laser and Doppler tracking [17], and they are slowly building up with time (see Figure 2 and Figure 3), as the spectral radius $\rho$ is larger than one and $s\left(t_{0}+T\right)$ is non zero (we have $\delta r\left(t_{0}+T\right)=1.12 \mathrm{~cm}$ and $\delta v\left(t_{0}+T\right)=4.9 \times 10^{-3} \mathrm{~mm} / \mathrm{s}$ ). A strategy to look at these perturbations would be to put a LAGEOS-like satellite with laser cubes [18] in such an orbit, and to observe it during

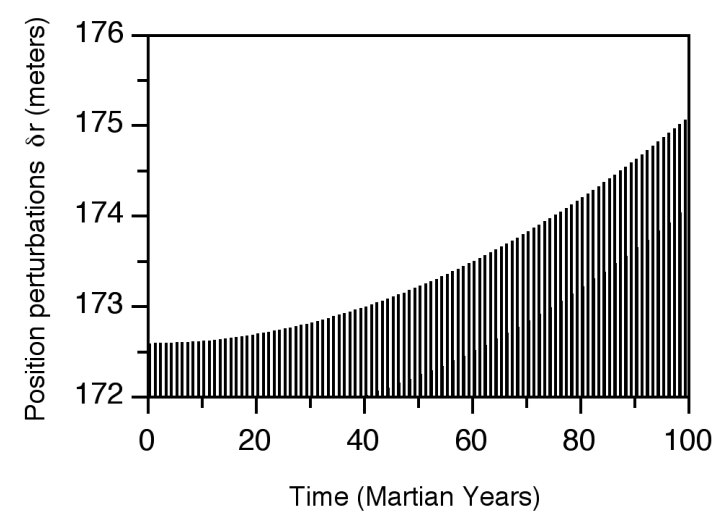

Figure 2. Building up of the position perturbations $\delta r$ over the Martian years in meters. The building up of the perturbations is not strictly periodic (with a shift of about 14 minutes/year), albeit the excitation mechanism is by itself periodic. 


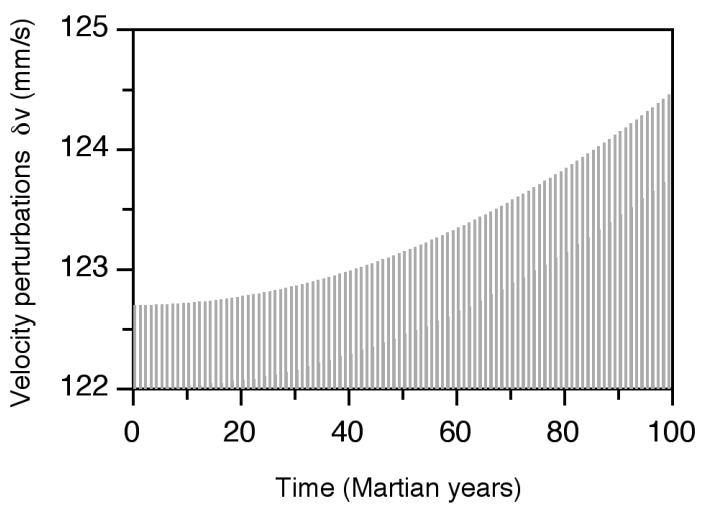

Figure 3. Building up of the velocity perturbations $\delta v$ over the years in $\mathrm{mm} / \mathrm{second}$.

a sufficient amount of time, from other Mars satellites, or even from the Earth, if it is equipped with "active" laser receptors [19] instead of passive retroreflectors.

We believe that this phenomenon is general, and that the theory described in this paper deserves to be generalized to any type of orbit, including polar orbits dedicated to mapping. The analysis will be then complicated by the presence of the secular perturbations caused by the even zonal coefficients of the gravity field, and other long period perturbations. The effect of the perturbations that originate from the triaxiality of Mars is investigated in Appendix B. We plan also to study semiannual variations of the $J_{2}$ gravity coefficient, and to understand how the excitation mechanism described in this paper acts on the orbits of Phobos and Deimos that are near circular equatorial orbits.

\section{Acknowledgements}

This research was funded by the Centre National d'Etudes Spatiales (CNES).

\section{References}

[1] Chao, B.F. and Rubincam, D.P. (1990) Variations of Mars Gravitational Field and Rotation Due to Seasonal $\mathrm{CO}_{2} \mathrm{Ex}^{-}$ changes. Journal of Geophysical Research, 95, 14755-14760. http://dx.doi.org/10.1029/JB095iB09p14755

[2] Karatekin, O., Duron, J., Rosenblatt, P., Van Hoolst, T., Dehant, V. and Barriot, J.P. (2005) Mar's Time-Variable Gravity and Its Determination: Simulated Geodesy Experiments. Journal of Geophysical Research—Planets, 110, E06001. http://dx.doi.org/10.1029/2004JE002378

[3] Mioc, V. and Stavinschi, M. (2004) Stability of Satellite Orbits around Nonspherical Planets. Artificial Satellites, 39, 129-133.

[4] Jezewski, D.J. (1983) A Noncanonical Analytic Solution to the $\mathrm{J}_{2}$ Perturbed Two-Body Problem. Celestial Mechanics, 30, 343-361. http://dx.doi.org/10.1007/BF01375505

[5] Jezewski, D.J. (1983) An Analytical Solution for the $\mathrm{J}_{2}$ Perturbed Equatorial Orbit. Celestial Mechanics, 30, $363-371$. http://dx.doi.org/10.1007/BF01375506

[6] Chazy, J. (1953) Mécanique Céleste. Presses Universitaires de France, Paris.

[7] Dieudonné, J. (1980) Calcul Infinitésimal. Hermann Ed., Paris.

[8] Angot, A. (1972) Compléments de Mathématiques. Masson et Cie Ed., Paris.

[9] Walter, W. (1998) Ordinary Differential Equations. Springer, New-York.

[10] Roseau, M. (1976) Equations différentielles. Masson, Paris.

[11] Vijayaraghavan, A. (1984) An Analytic Solution for the Orbital Perturbations of the Venus Radar Mapper Due to Gravitational Harmonics. AIAA/AAS Astrodynamics Conference, Paper AIAA-84-1995.

[12] Mioc, V. and Stavinschi, M. (1998) Stability of Satellite Motion in the Equatorial Plane of the Rotating Earth. Proceedings of the Journées des Systèmes de Référence Spatio-Temporels, N. Capitaine Ed., 257-261.

[13] Mioc, V. and Stavinschi, M. (2001) Effects of Mars’ Rotation on Orbiter Dynamics. Proceedings of the Journées des Systèmes de Référence Spatio-Temporels, N. Capitaine Ed, 120-125. 
[14] Mioc, V. and Stavinschi, M. (2003) Stability of Equatorial Satellite Orbits. Proceedings of the Journées des Systèmes de Référence Spatio-Temporels, N. Capitaine Ed., 255-258.

[15] Folkner, W.M., Yoder, C.F., Yuan, D.N., Standish, E.M. and Preston, R.A. (1997) Interior Structure and Seasonal Mass Redistribution of Mars from Radio Tracking of Mars Pathfinder. Science, 278, 1749-1752. http://dx.doi.org/10.1126/science.278.5344.1749

[16] Lemoine, F.G., Smith, D.E., Rowlands, D.D., Zuber, M.T., Neumann, G.A., Chinn, D.S. and Pavlis, D.E. (2001) An Improved Solution of the Gravity Field of Mars (GMM-2B) from Mars Global Surveyor. Journal of Geophysical Research, 106, 23359-23376. http://dx.doi.org/10.1029/2000JE001426

[17] Moyer, T.D. (2000) Formulation for Observed and Computed Values of Deep Space Network Data Types for Navigation. Monograph 2, Deep Space Communications and Navigation Series, JPL Publication 00-7.

[18] Yoder, C.F., Williams, J.G., Dickey, J.O., Schutz, B.E., Eanes, R.J. and Tapley, B.D. (1983) Secular Variation of Earth's Gravitational Harmonic $\mathrm{J}_{2}$ Coefficient from LAGEOS and Non-Tidal Acceleration of Earth's Rotation. Nature, 303, 757-762. http://dx.doi.org/10.1038/303757a0

[19] Samain, E. (2002) One Way Laser Ranging on the Solar System: TIPO. Geophysical Research Abstracts, 4, Article ID: 05808.

[20] Hu, W. and Scheeres, D.J. (2004) Numerical Determination of Stability Regions for Orbital Motion in Uniformly Rotating Second Degree and Order Gravity Fields. Planetary and Space Science, 52, 685-692. http://dx.doi.org/10.1016/j.pss.2004.01.003 


\section{Appendix A}

We have, with $t_{0} \leq t<T$, by using Floquet's theory,

$$
\begin{aligned}
s(t+k T) & =\int_{t_{0}}^{t+k T} \Pi(t+k T, \xi) w(\xi) \mathrm{d} \xi \\
& =\int_{t_{0}}^{t+k T} \Pi\left(t+k T, t_{0}+T\right) \Pi\left(t_{0}+T, \xi\right) w(\xi) \mathrm{d} \xi \\
& =\Pi\left(t, t_{0}\right) C^{k-1} \int_{t_{0}}^{t+k T} \Pi\left(t_{0}+T, \xi\right) w(\xi) \mathrm{d} \xi \\
& =\Pi\left(t, t_{0}\right) C^{k-1}\left(\sum_{j=0}^{j=k-1} C^{-j} \int_{t_{0}}^{t_{0}+T} \Pi\left(t_{0}+T, \xi\right) w(\xi) \mathrm{d} \xi+\Pi\left(t, t_{0}\right) C^{-1} \int_{t_{0}}^{t} \Pi\left(t_{0}+T, \xi\right) w(\xi) \mathrm{d} \xi\right. \\
& =\Pi\left(t, t_{0}\right)\left(\sum_{j=0}^{j=k-1} C^{j} \int^{t_{0}+T} \int_{t_{0}}^{t} \Pi\left(t_{0}+T, \xi\right) w(\xi) \mathrm{d} \xi+\Pi\left(t, t_{0}+T\right) \int_{t_{0}}^{t} \Pi\left(t_{0}+T, \xi\right) w(\xi) \mathrm{d} \xi\right. \\
& =\Pi\left(t, t_{0}\right) U^{(k)} \int_{t_{0}}^{t_{0}+T} \Pi\left(t_{0}+T, \xi\right) w(\xi) \mathrm{d} \xi+\int_{t_{0}}^{t} \Pi(t, \xi) w(\xi) \mathrm{d} \xi
\end{aligned}
$$

with $U^{(k)}=\left(\sum_{j=0}^{j=k-1} C^{j}\right)=(I-C)^{-1}\left(I-C^{k}\right)$.

Therefore, $s(t+k T)=s(t)+\Pi\left(t, t_{0}\right) U^{(k)} s\left(t_{0}+T\right)$ with $t_{0} \leq t<t_{0}+T, \quad k \geq 0$,

$U^{(k)}=(I-C)^{-1}\left(I-C^{k}\right)=I+C U^{(k-1)}$, and $U^{(0)}=0, U^{(1)}=I$. In particular, if $t=t_{0}$, we have $s\left(t_{0}+k T\right)=U^{(k)} s\left(t_{0}+T\right)$.

Let us verify that this formula defines a continuous mapping of $s(t)$.

For $t=t_{0}+T-\varepsilon, \varepsilon \rightarrow 0, \varepsilon>0$ we have

$$
\begin{aligned}
s(t+k T) & \rightarrow s\left(t_{0}+T\right)+\Pi\left(t_{0}+T, t_{0}\right) U^{(k)} s\left(t_{0}+T\right) \\
& \rightarrow\left(I+C U^{(k)}\right) s\left(t_{0}+T\right)=U^{(k+1)} s\left(t_{0}+T\right) .
\end{aligned}
$$

For $t=t_{0}+T+\varepsilon, \varepsilon \rightarrow 0, \varepsilon>0$ we have

$$
\begin{aligned}
s(t+k T) & \rightarrow s\left(t_{0}\right)+\Pi\left(t_{0}, t_{0}\right) U^{(k+1)} s\left(t_{0}+T\right) \\
& \rightarrow U^{(k+1)} s\left(t_{0}+T\right),
\end{aligned}
$$

thus proving the continuity.

\section{Appendix B}

The above analysis supposes that the equatorial moments of Mars are equal. Unfortunately, because of the Tharsis uplift, Mars is the terrestrial planet for which this assumption is the least accurate. If we take into account this triaxiality, the equations of motion (1) become, in an ad'hoc reference frame and up to degree and order two [20]

$$
\begin{aligned}
& \ddot{x}=-\frac{\mu}{d^{3}} x-J \frac{x}{d^{5}}+3 \mu R^{2} \kappa\left(\left(7 y^{2}-3 x^{2}\right) \frac{x}{d^{7}} \cos 2 \Theta+2\left(y^{2}-4 x^{2}\right) \frac{y}{d^{7}} \sin 2 \Theta\right) \\
& \ddot{y}=-\frac{\mu}{d^{3}} y-J \frac{y}{d^{5}}+3 \mu R^{2} \kappa\left(2\left(x^{2}-4 y^{2}\right) \frac{x}{d^{7}} \sin 2 \Theta-\left(7 x^{2}-3 y^{2}\right) \frac{y}{d^{7}} \cos 2 \Theta\right)
\end{aligned}
$$

where $\kappa=-6.3173 \times 10^{-5}$ in the system of constants of paragraph 5 and $\Theta=\Omega_{0} t-\frac{2}{3} \Delta \frac{M R^{2}}{C_{0}} \frac{\Omega_{0}}{\omega} \sin (\omega t+\varpi)+\Theta_{0}$. The Equations (9) can be then developed in Poincaré's series (see Eq- 
uation (3)), with respect to both $\Delta$ and $\kappa$. Up to the second order, and remembering that $\Delta=-1.8971 \times 10^{-9}$, we obtain $\kappa^{2}=4 \times 10^{-9}, \Delta^{2}=3.6 \times 10^{-18}$, and $\kappa \Delta=1.2 \times 10^{-13}$. This show that the excitation mechanism described in this paper is superimposed on the motion described by Equation (9) with $J_{2}(t)=J_{2}^{0}$ and $\Theta=\Omega_{0} t+\Theta_{0}$ up to degree one in $\Delta$ and degree two in $\kappa$. To be complete, all the other harmonic coefficients of the gravity field could be treated in the same way, provided that the circular equilibrium orbit is computed with respect to all even zonal coefficients. 